\title{
Minimax Theorems for Extended Real-Valued Abstract Convex-Concave Functions
}

\author{
Monika Syga $^{1}$ (D)
}

Received: 3 October 2016 / Accepted: 15 December 2017 / Published online: 27 December 2017

C The Author(s) 2017. This article is an open access publication

\begin{abstract}
In this paper, we provide sufficient and necessary conditions for the minimax equality for extended real-valued abstract convex-concave functions. As an application, we get sufficient and necessary conditions for the minimax equality for extended real-valued convex-concave functions.
\end{abstract}

Keywords Abstract convexity $\cdot$ Extended $\Phi$-convex functions $\cdot$ Minimax theorems

Mathematics Subject Classification 32F17 - 49J52 - 49K27 · 49K35 · 52A01

\section{Introduction}

Abstract convexity is a tool for global optimization. Abstract convex functions are defined as pointwise suprema of a given class of functions. This concept was first introduced by Moreau [1] and developed by Dolecki and Kurcyusz [2]. The monographs of Rubinov [3] and Rolewicz-Pallaschke [4] are devoted to this topic and discuss basic constructions of abstract convexity, like abstract conjugacy and abstract subdifferential, together with applications in optimization and numbers of important examples. In the present paper, we provide minimax theorems for functions, which are abstract convex with respect to one variable and concave with respect to the second variable.

Communicated by Juan-Enrique Martinez Legaz.

$\triangle \quad$ Monika Syga

M.Syga@mini.pw.edu.pl

1 Faculty of Mathematics and Information Science, Warsaw University of Technology, ul. Koszykowa 75, 00-662 Warsaw, Poland 
The first minimax theorem was proved by von Neumann in [5]. Since then there have been many generalizations of the original result, an exhaustive survey is given, e.g., in [6]; see also [7]. To the best of our knowledge, the existing minimax theorems are restricted to real-valued functions. Extended real-valued functions appear in standard constructions in variational analysis. It is a natural question what are the conditions under which the minimax equality for such functions holds. In this paper, we use the tools developed in [8] and [9] to provide minimax theorems for extended real-valued abstract convex-concave functions.

\section{Preliminaries}

We provide sufficient and necessary conditions for the minimax equality

$$
\sup _{y \in Y} \inf _{x \in X} a(x, y)=\inf _{x \in X} \sup _{y \in Y} a(x, y)
$$

where $X$ is nonempty set, $Y$ is a real vector space and $a: X \times Y \rightarrow \overline{\mathbb{R}}:=\mathbb{R} \cup\{ \pm \infty\}$ is an extended real-valued $\Phi$-convex (abstract convex) function with respect to $x$ and concave with respect to $y$.

Now, we recall some definitions related to $\Phi$-convexity. For any $f, g: X \rightarrow \overline{\mathbb{R}}$, $f \leq g$ if and only if $f(x) \leq g(x)$ for all $x \in X$.

Let $\Phi$ be defined as follows

$$
\Phi:=\{\ell+c, \quad \ell \in L, c \in \mathbb{R}\}
$$

where $L$ is an arbitrary class of functions $\ell: X \rightarrow \overline{\mathbb{R}}$. The class $L$ is called a set of abstract linear functions. The class $\Phi$ is called a set of abstract affine functions if it is stable by adding constants. Note that, if $L$ is the set of real-valued linear functions defined on $X$, then $\Phi$ is the set of real-valued affine functions defined on $X$.

The set supp $f \subset \Phi$, defined as

$$
\operatorname{supp} f:=\{\varphi \in \Phi: \varphi \leq f\}
$$

is called the support of $f$ with respect to $\Phi$. A function $f: X \rightarrow \overline{\mathbb{R}}$ is called $\Phi$-convex if

$$
\forall x \in X \quad f(x)=\sup \{\varphi(x): \varphi \in \operatorname{supp} f\} .
$$

A function $f: X \rightarrow \overline{\mathbb{R}}$ is proper if its effective domain is nonempty, i.e.,

$$
\operatorname{dom} f:=\{x \in X: f(x)<+\infty\} \neq \varnothing
$$

and $f(x)>-\infty$ for all $x \in X$. The set epi $f:=\{(x, r) \in X \times \mathbb{R}: r \geq f(x)\}$ is called the epigraph of $f$.

Let us note that a $\Phi$-convex function, taking the value $-\infty$, has an empty support or must contain in its support functions admitting $-\infty$. In the case where, $\Phi$ contains 
only real-valued functions $\varphi: X \rightarrow \mathbb{R}$, the $\Phi$-convex function $f: X \rightarrow \overline{\mathbb{R}}$ is proper, if and only if supp $f \neq \varnothing$ and $\operatorname{dom} f \neq \emptyset$.

For any $\varphi: X \rightarrow \overline{\mathbb{R}}$ and $Z \subset X$ we define the strict lower level set of $\varphi$ at the level $\alpha \in \mathbb{R}$ as

$$
[\varphi<\alpha]_{Z}:=\{x \in Z: \varphi(x)<\alpha\},
$$

if $Z=X$, then we use the notation $[\varphi<\alpha]_{X}:=[\varphi<\alpha]$.

Minimax theorems for functions $a: X \times Y \rightarrow \mathbb{R} \cup\{+\infty\}$, where for each $y \in Y$ the function $a(\cdot, y): X \rightarrow \mathbb{R} \cup\{+\infty\}$ is $\Phi$-convex, are based on the following intersection property introduced in [8] and investigated in [10] and [9].

Definition 2.1 Let $\varphi_{1}, \varphi_{2}: X \rightarrow \mathbb{R}$ be any two functions and $\alpha \in \mathbb{R}$. The functions $\varphi_{1}$ and $\varphi_{2}$ are said to have the intersection property on $X$ at the level $\alpha \in \mathbb{R}$ iff for every $t \in[0,1]$

$$
\left[t \varphi_{1}+(1-t) \varphi_{2}<\alpha\right] \cap\left[\varphi_{1}<\alpha\right]=\emptyset \text { or }\left[t \varphi_{1}+(1-t) \varphi_{2}<\alpha\right] \cap\left[\varphi_{2}<\alpha\right]=\emptyset .
$$

Remark 2.1 Functions $\varphi_{1}, \varphi_{2}: X \rightarrow \mathbb{R}$, which belong to the class

$\Phi_{l s c}:=\left\{\varphi: X \rightarrow \mathbb{R}, \varphi(x)=-a\|x\|^{2}+\langle\ell, x\rangle+c, \quad x \in X, \ell \in X^{*}, a \geq 0, c \in \mathbb{R}\right\}$,

where $X$ is a normed space, and $X^{*}$ is a topological dual to $X$, have the intersection property on $X$ at the level $\alpha$ if and only if

$$
\left[\varphi_{1}<\alpha\right] \cap\left[\varphi_{2}<\alpha\right]=\emptyset
$$

For more results along this line see [9].

Let us note that by Rubinov's theorem ([3], Example 6.6) a proper lower semicontinuous function $f: X \rightarrow \mathbb{R} \cup\{+\infty\}$ defined on Hilbert space $X$ is $\Phi_{\text {lsc }}$-convex if there exists $\bar{\varphi} \in \Phi_{\text {lsc }}$ such that $\bar{\varphi}<f$.

The following theorem has been proved in [10].

Theorem 2.1 (Theorem 3.3.3 of [10]) Let $X$ be a set and $Y$ be a real vector space. Let $\Phi$ be a class of real-valued functions $\varphi: X \rightarrow \mathbb{R}$ which contains the constant functions. Let $a: X \times Y \rightarrow \mathbb{R} \cup\{+\infty\}$ be a function such that

- for any $y \in Y$ the function $a(\cdot, y): X \rightarrow \mathbb{R} \cup\{+\infty\}$ is $\Phi$-convex on $X$,

- for any $x \in X$ the function $a(x, \cdot): Y \rightarrow \mathbb{R} \cup\{+\infty\}$ is concave on $Y$.

The following conditions are equivalent:

(i) for every $\alpha \in \mathbb{R}, \alpha<\inf _{x \in X} \sup _{y \in Y} a(x, y)$, there exist $y_{1}, y_{2} \in Y$ and $\varphi_{1} \in \operatorname{supp} a\left(\cdot, y_{1}\right), \varphi_{2} \in \operatorname{supp} a\left(\cdot, y_{2}\right)$ such that the intersection property holds for $\varphi_{1}, \varphi_{2}$ on $X$ at the level $\alpha$.

(ii) $\sup _{y \in Y} \inf _{x \in X} a(x, y)=\inf _{x \in X} \sup _{y \in Y} a(x, y)$.

The proof of this fact will be given as a corollary of Theorem 5.1. 


\section{Generalized Intersection Property}

Throughout this paper, we use the following convention

$$
\begin{aligned}
& +\infty+(-\infty)=-\infty+(+\infty)=+\infty, \quad 0 \cdot(+\infty)=0, \quad 0 \cdot(-\infty)=0, \\
& \forall t \in] 0,1]: t \cdot(+\infty)=+\infty, \quad t \cdot(-\infty)=-\infty
\end{aligned}
$$

In [11], the above addition was called inf-addition (the authors considered also supaddition).

To derive necessary and sufficient conditions for the minimax equality for extended real-valued $\Phi$-convex-concave functions we use the generalized intersection property introduced below.

Definition 3.1 Let $\varphi_{1}, \varphi_{2}: X \rightarrow \overline{\mathbb{R}}$ be any two functions and $\alpha \in \mathbb{R}$. The functions $\varphi_{1}$ and $\varphi_{2}$ are said to have the generalized intersection property on $X$ at the level $\alpha \in \mathbb{R}$ iff (2) hold:

$$
\forall t \in[0,1]:\left[t \varphi_{1}+(1-t) \varphi_{2}<\alpha\right] \cap\left[\varphi_{1}<\alpha\right]=\emptyset \text { or }\left[t \varphi_{1}+(1-t) \varphi_{2}<\alpha\right] \cap\left[\varphi_{2}<\alpha\right]=\emptyset
$$

and (3) or (4) or (5) hold:

$$
\begin{aligned}
& {\left[\varphi_{1}<\alpha\right]=\varnothing} \\
& {\left[\varphi_{2}<\alpha\right]=\varnothing} \\
& \exists t \in] 0,1\left[: \quad\left[\varphi_{1}<\alpha\right] \cap\left[t \varphi_{1}+(1-t) \varphi_{2}<\alpha\right]=\emptyset \quad\right. \text { (a) } \\
& \exists t \in] 0,1\left[: \quad\left[\varphi_{2}<\alpha\right] \cap\left[t \varphi_{1}+(1-t) \varphi_{2}<\alpha\right]=\emptyset \quad\right. \text { (b) }
\end{aligned}
$$

Proposition 3.1 Let $\varphi_{1}, \varphi_{2}$ admit only finite values, i.e., $\varphi_{1}, \varphi_{2}: X \rightarrow \mathbb{R}$. Then, $\varphi_{1}, \varphi_{2}$ have the generalized intersection property on $X$ at the level $\alpha$ if and only if $\varphi_{1}, \varphi_{2}$ have the intersection property on $X$ at the level $\alpha$.

Proof Assume that $\varphi_{1}, \varphi_{2}$ have the intersection property on $X$ at the level $\alpha$.

It is obvious that (2) holds. Then, the following situations may occur:

1. $\left[\varphi_{1}<\alpha\right]=\emptyset$, hence (3) holds.

2. $\left[\varphi_{2}<\alpha\right]=\emptyset$, hence (4) holds.

3. $\left[\varphi_{1}<\alpha\right] \neq \varnothing$ and $\left[\varphi_{2}<\alpha\right] \neq \varnothing$. Then, for any $\bar{x} \in\left[\varphi_{1}<\alpha\right]$, we have $0 \leq F_{1}(\bar{x}):=\frac{\alpha-\varphi_{2}(\bar{x})}{\varphi_{1}(\bar{x})-\varphi_{2}(\bar{x})}<1$ and for any $\bar{t}, F_{1}(\bar{x})<\bar{t}<1$ we get

$$
\left[\varphi_{2}<\alpha\right] \cap\left[\bar{t} \varphi_{1}+(1-\bar{t}) \varphi_{2}<\alpha\right]=\emptyset,
$$

and (5)b holds. Analogously, for any $\bar{x} \in\left[\varphi_{2}<\alpha\right]$, we have $0<F_{2}(\bar{x}):=$ $\frac{\alpha-\varphi_{2}(\bar{x})}{\varphi_{1}(\bar{x})-\varphi_{2}(\bar{x})} \leq 1$, and for any $\bar{t}, 0<\bar{t}<F_{2}(\bar{x})$ we get

$$
\left[\varphi_{1}<\alpha\right] \cap\left[\bar{t} \varphi_{1}+(1-\bar{t}) \varphi_{2}<\alpha\right]=\emptyset,
$$

and (5)a holds. 
Remark 3.1 (a) If there exists $\bar{x} \in X$ such that $\varphi_{1}(\bar{x})=-\infty$ and $\varphi_{2}(\bar{x})<+\infty$, then $\left[\varphi_{1}<\alpha\right] \neq \varnothing$ for all $\alpha \in \mathbb{R}$ and

$$
\left.\left.\left[t \varphi_{1}+(1-t) \varphi_{2}<\alpha\right] \cap\left[\varphi_{1}<\alpha\right] \neq \varnothing \text { for } t \in\right] 0,1\right]
$$

If there exists $\tilde{x} \in X \operatorname{such}$ that $\varphi_{2}(\tilde{x})=-\infty$ and $\varphi_{1}(\tilde{x})<+\infty$, then $\left[\varphi_{2}<\alpha\right] \neq \emptyset$ for all $\alpha \in \mathbb{R}$ and

$$
\left[t \varphi_{1}+(1-t) \varphi_{2}<\alpha\right] \cap\left[\varphi_{2}<\alpha\right] \neq \varnothing \text { for } t \in[0,1[
$$

Hence, (2) does not hold.

(b) If $\left[t \varphi_{1}+(1-t) \varphi_{2}<\alpha\right]=\emptyset$ for every $\left.t \in\right] 0,1\left[\operatorname{or}\left[\varphi_{1}<\alpha\right]=\emptyset\right.$ and $\left[\varphi_{2}<\alpha\right]=\emptyset$, then $\varphi_{1}, \varphi_{2}$ have the generalized intersection property on $X$ at the level $\alpha$.

Below we give examples related to generalized intersection property.

Example 3.1 1. Let $\varphi_{1}, \varphi_{2}: \mathbb{R} \rightarrow \overline{\mathbb{R}}$ be defined as follows

$$
\begin{aligned}
& \varphi_{1}(x)= \begin{cases}-\infty & \text { if, } x<a_{1}, \\
c_{1} & \text { if, } x=a_{1}, \\
+\infty & \text { if, } x>a_{1},\end{cases} \\
& \varphi_{2}(x)= \begin{cases}-\infty & \text { if, } x>a_{2}, \\
c_{2} & \text { if, } x=a_{2}, \\
+\infty & \text { if, } x<a_{2},\end{cases}
\end{aligned}
$$

where $a_{1}, a_{2}, c_{1}, c_{2} \in \mathbb{R}, a_{1}<a_{2}$. It is easy to see that functions $\varphi_{1}, \varphi_{2}$ have the generalized intersection property at every level $\alpha \in \mathbb{R}$.

2. Let $\varphi_{1}, \varphi_{2}: \mathbb{R} \rightarrow \overline{\mathbb{R}}$ be defined as follows

$$
\begin{aligned}
& \varphi_{1}(x)= \begin{cases}-\infty & \text { if, } x<a_{1}, \\
c_{1} & \text { if, } x=a_{1}, \\
+\infty & \text { if, } x>a_{1},\end{cases} \\
& \varphi_{2}(x)= \begin{cases}-\infty & \text { if, } x>a_{2}, \\
c_{2} & \text { if, } x=a_{2}, \\
+\infty & \text { if, } x<a_{2},\end{cases}
\end{aligned}
$$

where $a_{1}, a_{2}, c_{1}, c_{2} \in \mathbb{R}, a_{1}>a_{2}$. It is easy to see that for $\varphi_{1}, \varphi_{2}$ condition (5) does not hold, thus functions $\varphi_{1}, \varphi_{2}$ do not have the generalized intersection property at any level $\alpha$.

\section{An Auxiliary Lemma}

Now we prove a lemma which is crucial for our results.

Lemma 4.1 Let $\varphi_{1}, \varphi_{2}: X \rightarrow \overline{\mathbb{R}}$ and $\alpha \in \mathbb{R}$. The functions $\varphi_{1}, \varphi_{2}$ have the generalized intersection property on $X$ at the level $\alpha$ if and only if there exists $t_{0} \in[0,1]$ such that

$$
t_{0} \varphi_{1}(x)+\left(1-t_{0}\right) \varphi_{2}(x) \geq \alpha \text { for all } x \in X .
$$

Proof Assume that $\varphi_{1}, \varphi_{2}$ have the generalized intersection property on $X$ at the level $\alpha$. If $\left[\varphi_{1}<\alpha\right]=\emptyset$, then $\varphi_{1}(x) \geq \alpha$ for all $x \in X$, thus, in view of convention adopted, 
(6) holds for $t_{0}=1$. If $\left[\varphi_{2}<\alpha\right]=\emptyset$, then $\varphi_{2}(x) \geq \alpha$ for $x \in X$, thus (6) holds for $t_{0}=0$.

Assume now that $\left[\varphi_{1}<\alpha\right]$ and $\left[\varphi_{2}<\alpha\right]$ are nonempty and define the sets $T_{1}, T_{2}$,

$$
\begin{aligned}
& T_{1}:=\{t \in] 0,1\left[:\left[\varphi_{1}<\alpha\right] \cap\left[t \varphi_{1}+(1-t) \varphi_{2}<\alpha\right] \neq \emptyset\right\} \\
& T_{2}:=\{t \in] 0,1\left[:\left[\varphi_{2}<\alpha\right] \cap\left[t \varphi_{1}+(1-t) \varphi_{2}<\alpha\right] \neq \emptyset\right\} .
\end{aligned}
$$

We show that $] 0,1\left[\backslash\left(T_{1} \cup T_{2}\right) \neq \emptyset\right.$. Consider the following cases:

1. $T_{1}=\emptyset$. By $\left.(5) \mathrm{b}, T_{2} \neq\right] 0,1[$.

2. $T_{2}=\emptyset$. Вy $\left.(5) \mathrm{a}, T_{1} \neq\right] 0,1[$.

3. $T_{1} \neq \emptyset, T_{2} \neq \emptyset$. We need to show that there exists $\left.t_{0} \in\right] 0,1\left[\backslash\left(T_{1} \cup T_{2}\right)\right.$. Observe that $T_{1} \cap T_{2}=\emptyset$. We show that $T_{1}$ and $T_{2}$ are open subsets of ]0, 1[. To show that $T_{1}$ is open, take any $\bar{t} \in T_{1}$, i.e., there exists $\bar{x} \in X$ such that

$$
\varphi_{1}(\bar{x})<\alpha \text { and } \bar{t} \varphi_{1}(\bar{x})+(1-\bar{t}) \varphi_{2}(\bar{x})<\alpha
$$

Clearly, it must be $\varphi_{1}(\bar{x})<+\infty$ and $\varphi_{2}(\bar{x})<+\infty$. By (5)a, it cannot be $\varphi_{1}(\bar{x})=-\infty$. Hence, $-\infty<\varphi_{1}(\bar{x})<+\infty$. By (2), it must be $\varphi_{2}(\bar{x})>-\infty$ since otherwise $\left[\varphi_{1}<\alpha\right] \cap\left[\varphi_{2}<\alpha\right] \neq \varnothing$. Let

$$
\varepsilon:=\frac{\alpha-\left(\bar{t} \varphi_{1}(\bar{x})+(1-\bar{t}) \varphi_{2}(\bar{x})\right)}{\varphi_{2}(\bar{x})-\varphi_{1}(\bar{x})}>0 .
$$

Then, for every $t \in[\bar{t}-\varepsilon, \bar{t}+\varepsilon]$, we have $t \in T_{1}$.

To show that $T_{2}$ is open, take any $\tilde{t} \in T_{2}$, i.e., there exists $\tilde{x} \in X$ such that

$$
\varphi_{2}(\tilde{x})<\alpha \text { and } \tilde{t} \varphi_{1}(\tilde{x})+(1-\tilde{t}) \varphi_{2}(\tilde{x})<\alpha .
$$

Clearly, $\varphi_{1}(\tilde{x})$ and $\varphi_{2}(\tilde{x})$ are finite. Let

$$
\varepsilon:=\frac{\alpha-\left(\tilde{t} \varphi_{1}(\tilde{x})+(1-\tilde{t}) \varphi_{2}(\tilde{x})\right)}{\varphi_{1}(\tilde{x})-\varphi_{2}(\tilde{x})}>0
$$

then for every $t \in[\tilde{t}-\varepsilon, \tilde{t}+\varepsilon]$ we have $t \in T_{2}$.

Since $T_{1}, T_{2}$ are nonempty and disjoint, and we showed that they are open, we get ] $0,1\left[\backslash\left(T_{1} \cup T_{2}\right) \neq \emptyset\right.$.

For all $\left.t_{0} \in\right] 0,1\left[\backslash\left(T_{1} \cup T_{2}\right)\right.$ we have

$$
t_{0} \varphi_{1}(x)+\left(1-t_{0}\right) \varphi_{2}(x) \geq \alpha \text { for all } x \in X .
$$

To show the converse implication, assume now that (6) holds. If $t_{0}=0$, then $\varphi_{2}(x) \geq \alpha$, for all $x \in X$, and conditions (2) and (4) of Definition 3.1 are fulfilled. If $t_{0}=1$, then $\varphi_{1}(x) \geq \alpha$, for all $x \in X$, and conditions (2) and (3) of Definition 3.1 hold. 
Consider now the case where (6) holds with $\left.t_{0} \in\right] 0,1[$. It is easy to see that (5) is true for $t_{0}$.

To complete the proof we need to show that condition (2) holds. Observe first that, by (6), it must be $\left[\varphi_{1}<\alpha\right] \cap\left[\varphi_{2}<\alpha\right]=\emptyset$, since otherwise there exists $\bar{x} \in\left[\varphi_{1}<\alpha\right] \cap\left[\varphi_{2}<\alpha\right]$ such that $t_{0} \varphi_{1}(\bar{x})+\left(1-t_{0}\right) \varphi_{2}(\bar{x}) \geq \alpha$.

By contradiction, suppose that $\left[\varphi_{1}<\alpha\right] \cap\left[\varphi_{2}<\alpha\right]=\emptyset$ and (2) does not hold, i.e., there exist $\left.t_{1} \in\right] 0,1[$ and $\bar{x}, \tilde{x} \in X$ such that

$$
\begin{aligned}
& \bar{x} \in\left[\varphi_{1}<\alpha\right] \cap\left[t_{1} \varphi_{1}+\left(1-t_{1}\right) \varphi_{2}<\alpha\right] \\
& \tilde{x} \in\left[\varphi_{2}<\alpha\right] \cap\left[t_{1} \varphi_{1}+\left(1-t_{1}\right) \varphi_{2}<\alpha\right] .
\end{aligned}
$$

Then

$$
\varphi_{1}(\bar{x})<\alpha, \quad \varphi_{2}(\bar{x}) \geq \alpha \quad \varphi_{2}(\tilde{x})<\alpha, \quad \varphi_{1}(\tilde{x}) \geq \alpha .
$$

Now we show that $\varphi_{1}(\bar{x}), \varphi_{2}(\bar{x}), \varphi_{1}(\tilde{x})$ and $\varphi_{2}(\tilde{x})$ are finite. Clearly $\varphi_{1}(\bar{x})<+\infty$, $\varphi_{1}(\tilde{x})>-\infty$ and $\varphi_{2}(\tilde{x})<+\infty, \varphi_{2}(\bar{x})>-\infty$. Moreover, by (7), it must be $\varphi_{1}(\tilde{x})<+\infty$ and $\varphi_{2}(\bar{x})<+\infty$; consequently, by (6) it must be $\varphi_{1}(\bar{x})>-\infty$ and $\varphi_{2}(\tilde{x})>-\infty$. By $(7)$,

$$
\frac{\alpha-\varphi_{2}(\bar{x})}{\varphi_{1}(\bar{x})-\varphi_{2}(\bar{x})}<t_{1}<\frac{\alpha-\varphi_{2}(\tilde{x})}{\varphi_{1}(\tilde{x})-\varphi_{2}(\tilde{x})},
$$

which contradicts (6).

\section{Main Results}

Now we are in a position to prove necessary and sufficient conditions for the minimax equality for extended real-valued $\Phi$-convex-concave functions.

We say that a function $f: X \rightarrow \overline{\mathbb{R}}$ is concave in the sense of Ky Fan [12] if for any $x_{1}, x_{2} \in X$ and $t \in[0,1]$ there exists $x_{0} \in X$ such that $f\left(x_{0}\right) \geq t f\left(x_{1}\right)+(1-t) f\left(x_{2}\right)$.

Theorem 5.1 Let $X$ be a set and $Y$ be a real vector space. Let $a: X \times Y \rightarrow \overline{\mathbb{R}}$,

$-\forall y \in Y, a(\cdot, y): X \rightarrow \overline{\mathbb{R}}$ is $\Phi$-convex on $X$,

$-\forall x \in X, a(x, \cdot): Y \rightarrow \overline{\mathbb{R}}$ is concave on $Y$.

If

(i) for every $\alpha \in \mathbb{R}, \alpha<\inf _{x \in X} \sup _{y \in Y} a(x, y)$, there exist $y_{1}, y_{2} \in Y$ and $\varphi_{1} \in \operatorname{supp} a\left(\cdot, y_{1}\right), \varphi_{2} \in \operatorname{supp} a\left(\cdot, y_{2}\right)$ such that the generalized intersection property holds for $\varphi_{1}, \varphi_{2}$ on $X$ at the level $\alpha$.

then

(ii) $\sup _{y \in Y} \inf _{x \in X} a(x, y)=\inf _{x \in X} \sup _{y \in Y} a(x, y)$.

Moreover, if $\Phi$ contains all constant functions, then (ii) $\Rightarrow(i)$. 
Proof (i) $\Rightarrow$ (ii) If $\inf _{x \in X} \sup _{y \in Y} a(x, y)=-\infty$, then by the fact that the inequality

$$
\sup _{y \in Y} \inf _{x \in X} a(x, y) \leq \inf _{x \in X} \sup _{y \in Y} a(x, y)
$$

always holds, we get the required conclusion.

Let $\alpha<\inf _{x \in X} \sup _{y \in Y} a(x, y)$. By Lemma 4.1, there exists $t_{0} \in[0,1]$ such that

$$
t_{0} \varphi_{1}(x)+\left(1-t_{0}\right) \varphi_{2}(x) \geq \alpha \text { for all } x \in X,
$$

where $\varphi_{1} \in \operatorname{supp} a\left(\cdot, y_{1}\right), \varphi_{2} \in \operatorname{supp} a\left(\cdot, y_{2}\right), y_{1}, y_{2} \in Y$. Hence

$$
t_{0} a\left(x, y_{1}\right)+\left(1-t_{0}\right) a\left(x, y_{2}\right) \geq \alpha \text { for all } x \in X
$$

By concavity of $a(x, \cdot)$ and by (9), there exists $y_{0} \in Y$ such that

$$
a\left(x, y_{0}\right) \geq \alpha \text { for all } x \in X \text {. }
$$

Thus

$$
\sup _{y \in Y} \inf _{x \in X} a(x, y) \geq \alpha
$$

for all $\alpha<\inf _{x \in X} \sup _{y \in Y} a(x, y)$. Hence, we get

$$
\sup _{y \in Y} \inf _{x \in X} a(x, y) \geq \inf _{x \in X} \sup _{y \in Y} a(x, y) .
$$

(ii) $\Rightarrow$ (i) We need only to consider the case $\inf _{x \in X} \sup _{y \in Y} a(x, y)>-\infty$. Let $\alpha<\inf _{x \in X} \sup _{y \in Y} a(x, y)$. By the equality, $\sup _{y \in Y} \inf _{x \in X} a(x, y)=\inf _{x \in X} \sup _{y \in Y}$ $a(x, y)$, we get

$$
\sup _{y \in Y} \inf _{x \in X} a(x, y)>\alpha .
$$

So, there exists $\bar{y} \in Y$ such that

$$
a(x, \bar{y}) \geq \alpha \text { for all } x \in X
$$

Thus, the function $\bar{\varphi}:=\alpha$ belongs to the support set supp $a(\cdot, \bar{y})$. By the fact that $[\bar{\varphi}<\alpha]=\emptyset$, we get

$$
[t \bar{\varphi}+(1-t) \varphi<\alpha] \cap[\bar{\varphi}<\alpha]=\emptyset \text { for all } \varphi \in \Phi
$$

Then, for all $\varphi \in \Phi$, the functions $\bar{\varphi}$ and $\varphi$ have the generalized intersection property on $X$ at the level $\alpha$. 
Proof of Theorem 2.1 The proof follows directly from Remark 3.1a and Theorem 5.1.

Let us note that in the proof of Theorem 5.1 the roles of $a(\cdot, y)$ and $a(x, \cdot)$ are not symmetric, i.e., one cannot get the conclusion of Theorem 5.1 under the assumption that $a(x, \cdot)$ is $\Psi$-concave for a certain class $\Psi$.

By examining the proof of Theorem 5.1, we see that the fact, that functions $a(\cdot, y)$ are pointwise suprema of functions from $\Phi$ is not used. What is needed in the proof is that, for $\alpha<\inf _{x \in X} \sup _{y \in Y} a(x, y)$ there exist $y_{1}, y_{2} \in Y$ and any functions $\varphi_{1}, \varphi_{2}: X \rightarrow \overline{\mathbb{R}}, \varphi_{1} \leq a\left(\cdot, y_{1}\right), \varphi_{2} \leq a\left(\cdot, y_{2}\right)$, satisfying the generalized intersection property on $X$ at the level $\alpha$.

This allows to formulate the following theorem.

Theorem 5.2 Let $X$ be a set and $Y$ be a real vector space.

Let $a: X \times Y \rightarrow \overline{\mathbb{R}}$ be such that:

- for any $x \in X$ the function $a(x, \cdot): Y \rightarrow \overline{\mathbb{R}}$ is concave on $Y$.

The following conditions are equivalent:

(i) for every $\alpha \in \mathbb{R}, \alpha<\inf _{x \in X} \sup _{y \in Y} a(x, y)$, there exist $y_{1}, y_{2} \in Y$ and

$\varphi_{1} \in \operatorname{supp} a\left(\cdot, y_{1}\right), \varphi_{2} \in \operatorname{supp} a\left(\cdot, y_{2}\right)$ such that the generalized intersection property holds for $\varphi_{1}, \varphi_{2}$ on $X$ at the level $\alpha$.

(ii) $\sup _{y \in Y} \inf _{x \in X} a(x, y)=\inf _{x \in X} \sup _{y \in Y} a(x, y)$.

Proof The same as the proof of Theorem 5.1.

Remark 5.1 Let $X, Y, \Phi$ and $a(\cdot, \cdot)$ be as in Theorem 5.1 .

1. If there exists $\bar{y} \in Y$ such that $a(\cdot, \bar{y}) \equiv+\infty$, then $\inf _{x \in X} a(x, \bar{y})=+\infty$, hence $\sup _{y \in Y} \inf _{x \in X} a(x, y)=+\infty$. On the other hand $\sup _{y \in Y} a(x, y)=+\infty$, for every $x \in X$, so inf $x \in X \sup _{y \in Y} a(x, y)=+\infty$ and minimax equality always holds for function $a(\cdot, \cdot)$.

2. If there exists $\bar{x} \in X$ such that $a(\bar{x}, \cdot) \equiv-\infty$, then $\inf _{x \in X} \sup _{y \in Y} a(x, y)=-\infty$, and condition $(i)$ of Theorem 5.1 always holds.

In Theorem 5.1 we can change the assumptions on function $a(\cdot, \cdot)$ symmetrically. It is possible to assume that $a(\cdot, \cdot)$ is convex as a function of $X$ and $\Phi$-concave as a function of $y$, i.e., is equal to the pointwise infimum of all functions $\varphi$ grater than or equal to $a(\cdot, \cdot)$. In such case condition $(i)$ of Theorem 5.1 has to hold for all $\alpha>\sup _{y \in Y} \inf _{x \in X} a(x, y)$ and definition of generalized intersection property with opposite inequalities.

\section{$6 \Phi$-convexity of Convex Functions}

In this section, we present a class $\Phi$ such that all convex functions defined on $\mathbb{R}^{n}$ are $\Phi$-convex (Theorem 6.1). This class is used in next section to provide minimax theorem for convex-concave functions. 
We recall that $x=\left(x^{1}, \ldots, x^{n}\right)^{T} \in \mathbb{R}^{n}$ is said to be "lexicographically less" than $y=\left(y^{1}, \ldots, y^{n}\right)^{T} \in \mathbb{R}^{n}$, denoted $x<_{L} y$, if $x \neq y$ and for

$k=\min \left\{i \in\{1, \ldots, n\} \mid x_{i} \neq y_{i}\right\}$ we have $x_{k}<y_{k}$.

For $k \in\{0,1, \ldots, n\}$, we denote by $\mathcal{L}\left(\mathbb{R}^{n}, \mathbb{R}^{k}\right)$ the set of all linear mappings $u: \mathbb{R}^{n} \rightarrow \mathbb{R}^{k}$.

Following [13], for any $u \in \mathcal{L}\left(\mathbb{R}^{n}, \mathbb{R}^{k}\right)$, with rank $u=k, z \in \mathbb{R}^{k}, x^{*} \in\left(\mathbb{R}^{n}\right)^{*}$ and $d \in \mathbb{R}$, we define $\varphi_{u, z, x^{*}, d}: \mathbb{R}^{n} \rightarrow \overline{\mathbb{R}}$ by

$$
\varphi_{u, z, x^{*}, d}(x)=\left\{\begin{array}{lll}
-\infty & \text { if, } & u(x)<_{L} z \\
\left\langle x^{*}, x\right\rangle+d & \text { if, } & u(x)=z \\
+\infty & \text { if, } & u(x)>_{L} z
\end{array}\right.
$$

and let $\bar{\Phi}=\left\{\varphi_{u, z, x^{*}, d}\right\}$ be a set of all functions $\varphi_{u, z, x^{*}, d}$ defined above.

A function $f: \mathbb{R}^{n} \rightarrow \overline{\mathbb{R}}$ is convex if epigraph of $f$ is a convex set in $\mathbb{R}^{n}$.

Theorem 6.1 (Theorem 2.1, [13]) Let $f: \mathbb{R}^{n} \rightarrow \overline{\mathbb{R}}$. The following statements are equivalent

(i) $f$ is convex

(ii) $f$ is $\bar{\Phi}$-convex.

In the example, below we investigate functions $\varphi_{1}, \varphi_{2}: \mathbb{R} \rightarrow \overline{\mathbb{R}}, \varphi_{1}, \varphi_{2} \in \bar{\Phi}$ which have the generalized intersection property on $\mathbb{R}$ at the given level $\alpha$.

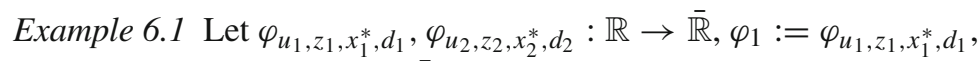

$\varphi_{2}:=\varphi_{u_{2}, z_{2}, x_{2}^{*}, d_{2}}, \varphi_{1}, \varphi_{2} \in \bar{\Phi}, \alpha \in \mathbb{R}$. In this setting we have $k=0$ or $k=1$, then either $u_{1} \equiv 0$ or $u_{1}$ is linear function from $\mathbb{R}$ to $\mathbb{R}$.

1. $u_{1} \equiv 0$.

(a) If $z_{1}>0$, then $\varphi_{1} \equiv-\infty . \varphi_{1}$ and $\varphi_{2}$ have the generalized intersection property if and only if $u_{2} \equiv 0$ and $z_{2}<0$, i.e., $\varphi_{2} \equiv+\infty$.

(b) If $z_{1}<0$, then $\varphi_{1} \equiv+\infty$. Thus, $\varphi_{1}$ and $\varphi_{2}$ have the generalized intersection property for every $u_{2}, z_{2}, x_{2}^{*}, d_{2}$.

(c) If $z_{1}=0$, then $\varphi_{1}$ is affine function. Thus, $\varphi_{1}$ and $\varphi_{2}$ have the generalized intersection property if and only if $u_{2} \equiv 0, z_{2}=0$ and $x_{1}^{*}, d_{1}, x_{2}^{*}, d_{2}$ are such that $\left[\varphi_{1}<\alpha\right] \cap\left[\varphi_{2}<\alpha\right]=\emptyset$.

2. $u_{1}(x)=a_{1} x, a_{1} \neq 0$ then $(\triangle)$ takes the form

$$
\varphi_{1}(x)= \begin{cases}-\infty & \text { if, } a_{1} x<z_{1} \\ \left\langle x_{1}^{*}, x\right\rangle+d_{1} & \text { if, } a_{1} x=z_{1} \\ +\infty & \text { if, } a_{1} x>z_{1}\end{cases}
$$

We have the following possible situations

(a) $u_{2} \equiv 0$, then $\varphi_{1}, \varphi_{2}$ have the generalized intersection property if and only if $z_{2}<0$, i.e., $\varphi_{2} \equiv+\infty$.

(b) $u_{2}(x)=a_{2} x, a_{2} \neq 0, a_{1}>0$, then $\varphi_{1}, \varphi_{2}$ have the generalized intersection property if and only if $a_{2}<0$ and $z_{1}<z_{2}$.

(c) $u_{2}(x)=a_{2} x, a_{2} \neq 0, a_{1}<0$, then $\varphi_{1}, \varphi_{2}$ have the generalized intersection property if and only if $a_{2}>0$ and $z_{1}>z_{2}$. 


\section{Minimax Theorems for Convex-Concave Functions}

Taking into account Theorem 6.1, we can formulate the minimax theorem for convexconcave functions.

Theorem 7.1 Let $Y$ be a real vector space. Let $a: \mathbb{R}^{n} \times Y \rightarrow \overline{\mathbb{R}}$ be such that

- for any $y \in Y$ the function $a(\cdot, y): \mathbb{R}^{n} \rightarrow \overline{\mathbb{R}}$ is convex on $\mathbb{R}^{n}$,

- for any $x \in \mathbb{R}^{n}$ the function $a(x, \cdot): Y \rightarrow \overline{\mathbb{R}}$ is concave on $Y$.

The following conditions are equivalent:

(i) for every $\alpha \in \mathbb{R}, \alpha<\inf _{x \in \mathbb{R}^{n}} \sup _{y \in Y} a(x, y)$, there exist $y_{1}, y_{2} \in Y$ and

$\varphi_{1} \in \operatorname{supp} a\left(\cdot, y_{1}\right), \varphi_{2} \in \operatorname{supp} a\left(\cdot, y_{2}\right)$ such that the generalized intersection property holds for $\varphi_{1}, \varphi_{2}$ on $\mathbb{R}^{n}$ at the level $\alpha$.

(ii) $\sup _{y \in Y} \inf _{x \in \mathbb{R}^{n}} a(x, y)=\inf _{x \in \mathbb{R}^{n}} \sup _{y \in Y} a(x, y)$.

Proof Follows from Theorems 6.1 and 5.1.

The following remark is based on Example 6.1.

Remark 7.1 Let $X, Y=\mathbb{R}$, and function $a(\cdot, \cdot)$ be as in Theorem 7.1.

1. If there exists $\bar{y} \in \mathbb{R}$ such that $a(\cdot, \bar{y}) \equiv+\infty$, then from Remark 5.1 we get that minimax equality holds,

2. If for every $y \in \mathbb{R}$ and every $x \in \mathbb{R}$ we have $a(x, y)<+\infty$, then for every $\varphi \in \operatorname{supp} a(\cdot, y)$ and every $x \in \mathbb{R}$ we have $\varphi(x)<+\infty$. Thus, for given level $\alpha \in \mathbb{R}$ there exist $y_{1}, y_{2} \in \mathbb{R}$ and $\varphi_{1} \in \operatorname{supp} a\left(\cdot, y_{1}\right), \varphi_{2} \in \operatorname{supp} a\left(\cdot, y_{2}\right)$ such that the generalized intersection property holds for $\varphi_{1}, \varphi_{2}$ on $\mathbb{R}$ at the level $\alpha$ if and only if $\varphi_{1}, \varphi_{2}$ are affine functions and $\left[\varphi_{1}<\alpha\right] \cap\left[\varphi_{2}<\alpha\right]=\emptyset$.

3. If there exist $\bar{y} \in \mathbb{R}$ and $\bar{x} \in \mathbb{R}$ such that $a(\bar{x}, \bar{y})=+\infty$ and $\tilde{x} \in X$ such that $a(\tilde{x}, \bar{y})<+\infty$, then all situations from Example 6.1 may occur.

4. If function $a(\cdot, \cdot)$ takes value $-\infty$, then all situations from Example 6.1 may occur.

We can formulate the following corollary of Theorem 7.1.

Corollary 7.1 Let $a: \mathbb{R} \times \mathbb{R} \rightarrow \overline{\mathbb{R}}$ be such that

- for every $y \in \mathbb{R}$ and every $x \in \mathbb{R} a(x, y)<+\infty$.

- for any $y \in \mathbb{R}$ the function $a(\cdot, y): \mathbb{R} \rightarrow \overline{\mathbb{R}}$ is convex on $\mathbb{R}$,

- for any $x \in \mathbb{R}$ the function $a(x, \cdot): \mathbb{R} \rightarrow \overline{\mathbb{R}}$ is concave on $\mathbb{R}$.

The following conditions are equivalent:

(i) for every $\alpha \in \mathbb{R}, \alpha<\inf _{x \in \mathbb{R}} \sup _{y \in \mathbb{R}} a(x, y)$, there exist $y_{1}, y_{2} \in \mathbb{R}$ and $\varphi_{1} \in \operatorname{supp} a\left(\cdot, y_{1}\right), \varphi_{2} \in \operatorname{supp} a\left(\cdot, y_{2}\right)$ such that $\varphi_{1}, \varphi_{2}$ are affine functions and $\left[\varphi_{1}<\alpha\right] \cap\left[\varphi_{2}<\alpha\right]=\emptyset$.

(ii) $\sup _{y \in \mathbb{R}} \inf _{x \in \mathbb{R}} a(x, y)=\inf _{x \in \mathbb{R}} \sup _{y \in \mathbb{R}} a(x, y)$.

Now, we present an example of improper convex-concave function $a(\cdot, \cdot)$, which dose not possess the generalized intersection property at any level $\alpha$. 
Example 7.1 Let $X, Y=\mathbb{R}$ and $a: \mathbb{R} \times \mathbb{R} \rightarrow \overline{\mathbb{R}}$.

$$
a(x, y)= \begin{cases}+\infty & \text { if, } x \geq 0, y<0 \text { or } x<0, y \geq 0 \\ -\infty & \text { if, } x \geq 0, y \geq 0 \text { or } x<0, y<0\end{cases}
$$

then $\inf _{x \in \mathbb{R}} \sup _{y \in \mathbb{R}} a(x, y)=+\infty$ and $\sup _{y \in \mathbb{R}} \inf _{x \in \mathbb{R}} a(x, y)=-\infty$. Then, for every $\alpha \in \mathbb{R}$ the generalized intersection property does not hold. Indeed, for $\bar{y} \geq 0$ function $a(\cdot, \bar{y})$ takes the form

$$
a(x, \bar{y})= \begin{cases}+\infty & \text { if, } x<0 \\ -\infty & \text { if, } x \geq 0\end{cases}
$$

and if $\varphi \in \operatorname{supp} a(\cdot, \bar{y})$, then $\varphi \equiv-\infty$.

For $\bar{y}<0$ function $a(\cdot, \bar{y})$ takes the form

$$
a(x, \bar{y})= \begin{cases}+\infty & \text { if, } x \geq 0 \\ -\infty & \text { if, } x<0\end{cases}
$$

and if $\varphi \in \operatorname{supp} a(\cdot, \bar{y})$, then

$$
\varphi(x)= \begin{cases}-\infty & \text { if, } x<0 \\ c & \text { if, } x=0 \\ +\infty & \text { if, } x>0\end{cases}
$$

where $c \in \mathbb{R}$.

\section{Conclusions}

We provide minimax theorems for extended real-valued $\Phi$-convex-concave functions (or symmetrically for convex- $\Phi$-concave functions). A distinguished feature of these results is that we do not need any topological structure on the spaces involved. On the other hand, the results obtained are applicable to functions being pointwise suprema of abstract affine functions. In particular, we obtain minimax theorems for extended real-valued convex-concave functions which do not have to be proper or lower (upper) semicontinuous.

Acknowledgements Author would like to thank Professor Jerzy Grzybowski from the University of Adam Mickiewicz in Poznan for suggesting in private communication that the equivalence in Lemma $4.1(\Leftarrow)$ may hold.

Open Access This article is distributed under the terms of the Creative Commons Attribution 4.0 International License (http://creativecommons.org/licenses/by/4.0/), which permits unrestricted use, distribution, and reproduction in any medium, provided you give appropriate credit to the original author(s) and the source, provide a link to the Creative Commons license, and indicate if changes were made. 


\section{References}

1. Moreau, J.J.: Inf-convolutions, sous-additivité, convexité des functions numériques. J. Math. pures et appl. 49, 109-154 (1970)

2. Dolecki, S., Kurcyusz, S.: On $\phi$-convexity in extremal problems. SIAM J. Control Optim. 16, 277-300 (1978)

3. Rubinov, A.M.: Abstract Convexity and Global Optimization. Kluwer Academic, Dordrecht (2000)

4. Pallaschke, D., Rolewicz, S.: Foundations of Mathematical Optimization. Kluwer Academic, Dordrecht (1997)

5. von Neumann, J.: Zur Theorie der Gesellschaftspiele. Math. Ann. 100, 295-320 (1928). English translation: On the theory of games of strategy. Contributions to the theory of games vol. 4, pp. 13-42. Princeton, Princeton. Univ. Press (1959)

6. Simons, S.: Minimax theorems and their proofs. Minimax and Applications pp. 1-23 (1995)

7. Greco, G.H., Horvath, C.D.: A topological minimax theorem. J. Optim. Theory Appl. 113, 513-536 (2002)

8. Bednarczuk, E.M., Syga, M.: Minimax theorems for $\phi$-convex functions with applications. Control Cybern. 43(3), 421-437 (2014)

9. Syga, M.: Minimax theorems for $\phi$-convex functions: sufficient and necessary conditions. Optimization 65(3), 635-649 (2016)

10. Syga, M.: Minimax theorems for $\phi$-convex functions with applications to optimization. In: Ph.D. thesis, Warsaw University of Technology, Faculty of Mathematics and Information Science, Warsaw. In Polish (2015)

11. Hamel, A.H., Schrage, C.: Notes about extended real- and set-valued functions. J. Convex Anal. 19, 355-384 (2012)

12. Fan, K.: Minimax theorems. Proc. Nat. Acad. Sci. 39, 42-47 (1953)

13. Martínez-Legaz, J.E., Singer, I.: On $\phi$-convexity of convex functions. Lin. Alg. Appl. 278, 163-181 (1998) 